\title{
Laboreal
}

Volume $1 \mathrm{~N}^{\circ} 1$ | 2005

Varia

\section{Aportes del método de los " puntos pivote " a un estudio prospectivo de seguridad en el campo de la interoperabilidad ferroviaria}

Contribuições do método dos "pontos-pivot" para um estudo prospectivo de segurança no campo da inter-operabilidade ferroviária

Apports de la méthode des "points-pivots" à une étude prospective de la sécurité sur le terrain de l'interopérabilité ferroviaire

Contributions of the "pivotal-points" method to a safety-related prospective study in the field of railway inter-operability

\section{Cecília De La Garza}

\section{OpenEdition}

Journals

Edición electrónica

URL: http://journals.openedition.org/laboreal/14006

DOI: $10.4000 /$ laboreal. 14006

ISSN: 1646-5237

Editor

Universidade do Porto

Referencia electrónica

Cecília De La Garza, "Aportes del método de los " puntos pivote " a un estudio prospectivo de seguridad en el campo de la interoperabilidad ferroviaria », Laboreal [En línea], Volume $1 N^{0} 1$ | 2005, Publicado el 01 diciembre 2005, consultado el 18 octubre 2019. URL : http://journals.openedition.org/ laboreal/14006 ; DOI : 10.4000/laboreal.14006

Este documento fue generado automáticamente el 18 octubre 2019

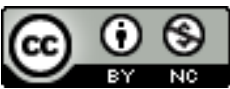

Laboreal está licenciado com uma Licença Creative Commons - Atribuição-NãoComercial 4.0 Internacional. 


\section{Aportes del método de los " puntos pivote " a un estudio prospectivo de seguridad en el campo de la interoperabilidad ferroviaria}

Contribuições do método dos "pontos-pivot" para um estudo prospectivo de segurança no campo da inter-operabilidade ferroviária

Apports de la méthode des "points-pivots" à une étude prospective de la sécurité sur le terrain de l'interopérabilité ferroviaire

Contributions of the "pivotal-points" method to a safety-related prospective study in the field of railway inter-operability

Cecília De La Garza

\section{NOTA DEL EDITOR}

Manuscrito recibido en : septiembre/2005

Aceptado tras peritage en : noviembre/2005

\section{Introducción : presentación del estudio de interoperabilidad ferroviaria}

Este estudio fue efectuado para la "Unión Internacional de Ferrocarriles" (Union Internationale des Chemins de fer UIC -). El objetivo final era el desarrollo de un método para el diseño de situaciones seguras y fiables de interoperabilidad tomando en cuenta los factores humanos. La interoperabilidad se define como la capacidad de circular indistintamente por cualquier sección de la red ferroviaria, sin detenerse en las fronteras y sin cambio de locomotora, ni de maquinista. Esto es la interoperabilidad 
"total", sin embargo en lo que los diferentes países logran una armonización en cuanto a la tecnología, material, normas, señalización, modos de frenado, corrientes de tracción, etc., se observan diferentes formas de interoperabilidad parcial en las fronteras como el cambio de maquinista sin cambio de locomotora o viceversa. La interoperabilidad es efectiva desde la apertura en marzo del 2003 de las fronteras europeas para el transporte de mercancías.

El estudio realizado para la UIC fue el resultado de un proyecto europeo organizado y efectuado por dos equipos de investigadores, uno en Francia y otro en Alemania [1].

Los resultados presentados aquí corresponden a un estudio de caso conducido por el equipo francés, basado en la utilización del método de los "puntos pivote". Esto es sólo una parte del estudio global el cual incluye además un análisis de la actividad laboral y del contexto de trabajo y una serie de encuestas y entrevistas sobre la experiencia de maquinistas habiendo practicado el paso de fronteras (De la Garza et al., 2004). En efecto, un estudio comparativo de dos centros de direccionamiento y del trayecto Woippy-Mannheim entre Francia y Alemania fue llevado a cabo entre septiembre del 2002 y marzo del 2003, durante la fase de implantación de la interoperabilidad. Dicho estudio permitió identificar los impactos tanto para los maquinistas como para el personal de un centro de direccionamiento, con la llegada de la interoperabilidad en relación con la formación, cambios en las tareas y en la carga del trabajo, sin contar los impactos sociales y otros (De la Garza, Kaplan \& Weill-Fassina, 2003). Para el maquinista se trata por ejemplo del aprendizaje de reglas de seguridad, de sistema de señalización de otro sistema ferroviario, de técnicas y estrategias de manejo, de idioma y lenguaje operativo etc.; en el ámbito social hay cambios en relación con el sueldo y la evolución en la empresa y la organización del tiempo laboral. Para los distintos operadores del centro de direccionamiento, según si éstos están en los puestos de control de las agujas, en los puestos de control del tráfico ferroviario o son agentes de maniobras, los cambios son varios : nueva documentación internacional, nuevos formularios, nuevas tareas de control de vagones, nuevas interacciones con los puestos del otro país para la gestión del tráfico, necesidad de sistemas de cómputo compatibles, necesidad de aprender un lenguaje operativo para una comunicación más fiable con maquinistas extranjeros, aumento de la carga de trabajo, etc. Se estableció así un diagnóstico de los puntos críticos y cambios que se deben controlar y anticipar para poder garantizar el funcionamiento fiable y eficaz del sistema organizacional futuro. Los resultados del análisis de los incidentes interoperables confirman y completan dichos puntos críticos como se verá en la parte siguiente.

El artículo se organiza como sigue : la presentación del objetivo del estudio de caso tratado aquí, un breve desarrollo del método de los puntos pivote que es aquí el principal instrumento metodológico, los resultados del análisis de 25 incidentes interoperables, la discusión y la conclusión.

\section{Objetivo : un análisis de tipo reactivo con un enfoque "proactivo"}

5 El objetivo de este articulo es el de mostrar cómo el método de los puntos-pivote, desarrollado para un análisis reactivo de accidentes e incidentes del trabajo, puede guiar la organización segura de una situación laboral. La distinción entre "reactivo" y "proactivo" es el momento en el que se efectúa el análisis y las recomendaciones : se 
trata aquí de integrar los riesgos en el diseño de la situación futura, buscando así evitar el surgimiento del evento crítico. El análisis de eventos críticos en situación de interoperabilidad, o cercana a la interoperabilidad, destaca elementos que permiten anticipar situaciones difíciles o peligrosas para un maquinista en el extranjero, orientando así la formación, como las barreras de seguridad necesarias a nivel de la organización. Se trata de eventos críticos en un centro de direccionamiento de trenes de mercancía o en una estación de tren de pasajeros : un descarrilamiento, una colisión lateral al engancharse en una aguja de manera forzada, pasarse una señal, o averiar material en la vía. Son incidentes interoperables ya que implican o a un maquinista extranjero, o una locomotora extranjera. Estos incidentes surgen al momento de la llegada, la partida o la preparación de un tren o durante las maniobras. Los actores implicados son los maquinistas y el personal del centro de direccionamiento.

\section{Presentación del método de los "puntos pivote" : bases teóricas y etapas}

6 Daremos aquí brevemente los fundamentos teóricos y las cuatro etapas del método de los "puntos pivote" que permiten una clasificación de los eventos críticos (De la Garza \& Weill-Fassina, 1995 ; De la Garza, 1999).

7 Desde el punto de vista práctico, el método fue desarrollado en el ámbito de la seguridad ferroviaria para analizar y comparar accidentes o incidentes graves o mortales. Diferentes aplicaciones han mostrado el interés de tal método como: el análisis de los accidentes que ocurren a los agentes de vías que efectúan operaciones de mantenimiento y que están expuestos al "riesgo de impacto" por circulación ferroviaria (De la Garza \& Weill-Fassina, 1995); el análisis de los incidentes por fallas de señalización engendradas por operaciones de mantenimiento de las instalaciones eléctricas (De la Garza, 1999) ; o el análisis de incidentes de los maquinistas ligados a la señalización u otros (Guyot-Delacroix, 2002).

El objetivo es estudiar y comparar las dinámicas de un grupo de eventos críticos. Se trata de reconstituir la lógica espacio-temporal de la génesis del accidente por medio de una confrontación de análisis de reportes, encuesta directa, testimonios y entrevistas con victimas y testigos, y cuando es posible, observaciones de campo.

\subsection{El vínculo entre un análisis individual y cognitivo y un análisis colectivo y organizacional de los eventos críticos}

Desde el punto de vista teórico, el método adopta un enfoque sistémico en donde el análisis se ubica en las interacciones entre los disfuncionamientos y las causas y elementos implicados en dicho evento. El método se apoya en el modelo del comportamiento de control del peligro de Hale \& Glendon (1987, véase anexo 1, en el fin del artículo) principalmente para el análisis cognitivo. Según este modelo, un disfuncionamiento puede ser controlado en diferentes etapas del procesamiento de la información : identificación, interpretación, evaluación, decisión y modos de acción. La hipótesis general es que todo proceso accidental o incidental resulta de interacciones no previstas en el proceso laboral, conduciendo a situaciones en las que los operadores no podrán responder adecuadamente, ni a tiempo, o no tendrán los medios de 
construirse un diagnóstico adaptado a la situación actual. Esta hipótesis se basa en ciertos modelos integrando la dinámica de los actores, así como la de la organización. Dichos modelos se enfocan en las restricciones de la organización ejerciendo presiones en el sistema y dan cuenta de las interacciones y de las condiciones de "migración" hacia umbrales de funcionamiento que se encuentran en el límite de la seguridad y de los resultados esperados (Rasmussen, 1997; Amalberti, 2001; Fadier, De la Garza \& Didelot, 2003).

El estudio de incidentes permite identificar por ejemplo, "fallas organizacionales", "errores de diseño" o bien "errores latentes" como elementos que favorecen el sobrepaso de barreras de seguridad (Reason, 1990 ; Leplat \& de Terssac, 1990 ; Perrow, 1984). Dicho método considera tanto la gestión individual del riesgo laboral, como una gestión colectiva de la seguridad que se inscribe en una red organizacional (De la Garza, 1999). En la medida en que se estudian "incidentes en situación de interoperabilidad", se considera que ciertas barreras de seguridad fueron ya sobrepasadas, pero no todas pues no se llegó al accidente. El estudio de estos eventos permite poner en relieve los factores propios a esta situación que contribuyeron de manera efectiva a una migración así como otros que aún no han sobrepasado los márgenes de seguridad, pero que están latentes o tienden a fragilizar la seguridad y la eficacia.

\subsection{Las cuatro etapas del método}

11 Cuatro etapas son necesarias en la aplicación del método de los puntos-pivote. Estas etapas se repiten cada vez que está uno confrontado a una nueva situación laboral u oficio.

\section{Establecer una tabla}

3 Esta tabla será común a todos los incidentes estudiados en un caso específico. Tiene por objetivo el lograr sintetizar los elementos de todos los incidentes a partir de una base común. Esta tabla incluye la organización laboral prevista, el entorno físico, climático y de infraestructura ferroviaria, la situación tal como estaba en el momento del evento crítico, las tareas, acciones y decisiones del personal del centro de direccionamiento, las del personal en los puestos de control del tráfico y las de los maquinistas (trenes y maniobras) (véase la primera columna del ejemplo de una historia de un incidente, tabla 1 en el fin del artículo). Esta tabla se construye a partir del análisis de casos, las encuestas con el personal y observaciones de campo de la actividad real.

\section{Reconstituir la historia del incidente}

5 En base a la tabla se reconstituye la historia de cada evento crítico. La historia describe la cronología de las acciones y decisiones, eventos en el tiempo y espacio. Para reconstituir la historia hay una doble entrada en la tabla : la columna de la izquierda que es una guía para saber que informaciones se buscan y se integran en el análisis, y la primera línea en la cual se van marcando los tiempos o momentos importantes del desarrollo del evento crítico (ver ejemplo tabla 1). Cada columna en la tabla 1 indica el surgimiento de un hecho o acción particular en el desarrollo del evento crítico. La historia se lee de izquierda a derecha, columna por columna. En general la última columna marca la manifestación de la consecuencia mayor del evento crítico. Sin embargo, una historia puede acumular varios incidentes como el del ejemplo de la tabla 1 . 
16 Desde luego que la objetividad y exactitud de cada uno de los elementos que constituyen cada incidente no se pueden validar completamente, ya que se trabaja a posteriori del evento crítico. Sin embargo el poder confrontar dicha información entre sí (reportes, entrevistas, observación de campo), es una manera de validar la historia. En el caso de los incidentes interoperables, falta información con relación a la organización prevista y su evolución antes del evento crítico, y con relación al tiempo (duración de un evento particular, momento en que un evento se produjo).

\section{Identificar y clasificar los puntos pivote en cada historia}

punto pivote se define como un elemento perturbador que, en interacción con otros puntos pivote y con el medio laboral, introduce un cambio de estado del sistema, el cual no podrá ser compensado. Los puntos pivote adquieren el estatus de "punto pivote" a posteriori, en función de las dificultades para el control de la situación por los actores implicados en el evento crítico y de las consecuencias en la dinámica de la situación. Así, la infracción de una regla podrá ser un punto pivote, pero no lo será de manera sistemática, ni será el único. El determinar los puntos pivote es una etapa fundamental del análisis ya que estos marcan los momentos de pérdida de control por parte de los actores en interacción con el entorno laboral. Por ejemplo aquí aparecerán casos de interpretación de omisión de transmisión de una información a un miembro del equipo, el olvido de una acción por carga excesiva de trabajo en un momento dado, el retardo de un tren y la ocupación de las vías que le corresponden, etc. Es así como se podrá establecer una tipología de los eventos críticos en la siguiente etapa.

\section{Establecer una tipología de eventos críticos}

La tipología de los eventos críticos se establece a través de una comparación de las historias reconstituidas. Dicha tipología describe categorías de secuencias de eventos y de acciones. Para esto se construye una tabla en donde se agrupan los puntos pivote de cada historia en relación con la etapa del proceso laboral en la cual surgieron y los actores implicados (véase tabla 2, cada columna representa un evento crítico en el fin del artículo). Esta categorización permite constituir un diagnóstico de los eventos críticos y por ende, proponer los fundamentos para una política de prevención englobando varios ámbitos.

\section{Resultados : veinticinco casos de incidentes interoperables analizados}

21 El estudio se realizó a partir de veinticinco casos que provienen de una base de datos interna a la empresa de ferrocarriles franceses DIESE (Datos Informáticos de la Experiencia y la Seguridad de la Explotación) ya que aún no existe una base de datos a nivel Europeo (Weill-Fassina, De la Garza \& Kaplan, 2003). DIESE es una base de datos nacional que integra los eventos críticos interoperables que se produjeron en las diferentes zonas fronterizas de Francia (Alemania, Bélgica, Italia, España, Luxemburgo). Así 23 eventos se produjeron en centros de direccionamiento y estaciones (Metz, Nancy, Bouzonville, Chambéry, Bordeaux, Montpellier...) y 2 durante un trayecto. Dichos eventos se produjeron entre enero del 1997 y febrero del 2003. Únicamente son colectados los eventos señalados por los centros franceses por lo tanto dicha base no es representativa de todos los eventos que se producen en las zonas fronterizas. Sin embargo los eventos estudiados aquí dan una idea bastante clara del tipo de problema 
que los maquinistas y actores de un centro de direccionamiento y estaciones deberán manejar de manera segura y eficaz.

Esta base de datos sólo provee una breve ficha por evento, sin un reporte detallado de la historia. Los elementos que constituyen la ficha Informática son los siguientes :

- Clasificación documento

- Identificación del evento

- Resumen del evento

- Causas identificadas (técnicas, factores humanos)

- Entorno geográfico

- Entorno técnico (descripción, causas, soluciones)

- Régimen de explotación

- Entorno físico

- Primera maniobra

- Características de evolución

- Segunda maniobra

- Organización respetada o no

- Factores humanos : primer agente, características, comportamiento, soluciones, clases de errores; segundo agente.

En esas fichas la interoperabilidad se refiere a un maquinista extranjero y/o a una locomotora extranjera. Sin embargo en algunos casos la nacionalidad de los maquinistas no fue mencionada y faltan otro tipo de informaciones como la cronología del evento. De hecho, sin un análisis de campo mediante observaciones de la actividad en un centro de direccionamiento y entrevistas con diferentes personales, este estudio no hubiese sido posible, ya que los datos almacenados en esas fichas son insuficientes y en ocasiones poco claros.

\subsection{La comunicación y la coordinación de las acciones como criterios de infiabilidad}

La tabla 1 muestra un incidente interoperable. La primera columna de la izquierda es la base común para el análisis de los 25 incidentes, es decir que en base a esa tabla cualquier evento crítico interoperable puede ser analizado. Según los casos, las diferentes secciones serán llenadas o no. Las columnas siguientes presentan de manera sintética el desarrollo del evento crítico. Los puntos pivote aparecen en tono de color distinto en relación con la taxonomía descrita aquí abajo.

Por ejemplo, en este caso los puntos pivote surgen en relación con una señal apagada, fallas en la coordinación de las acciones entre el personal del centro de control del tráfico.

Para poder interpretar los puntos pivote, explicarlos en el contexto particular de cada uno de los eventos críticos, el estudio se basó en una adaptación de la taxonomía propuesta por Hale \& Glendon (1987). A partir de esa taxonomía se puede interpretar en que momento surge la perturbación o pérdida de control de la situación (percepción, detección, decisión, etc.) y que factor o elemento de la situación está involucrado (organización, señal de alarma, tarea de rutina, etc.).

27 Para este estudio se utilizaron 5 categorías propuestas por Hale \& Glendon (1987) y se añade la del entorno climático, geográfico y físico que se ha utilizado de manera 
sistemática en las otras aplicaciones del método. Se presentan a continuación de manera detallada cada una de las categorías y se indica el color que le corresponde para facilitar la lectura y comprensión de los resultados presentados en la tabla 2.

- El control de la situación en las tareas de rutina. Las tareas de rutina son aquí las maniobras, la recepción de un tren o su partida involucrando a maquinistas y personal del centro de direccionamiento en los puestos de control del tráfico ferroviario y de las agujas y agentes de maniobras. Las dificultades resultan de procedimientos inadecuados durante la actividad laboral por alguno o varios de los actores, un incidente técnico o de otro orden que cambia el procedimiento habitual. La variabilidad de las tareas y entornos laborales son tales que se necesitan permanentemente ajustes cognitivos (en color café).

- El control en relación con la organización del trabajo y de la seguridad. Se trata aquí de identificar el control de acuerdo con los niveles jerárquicos y oficios involucrados en la organización prevista y los ajustes necesarios durante el desarrollo del proceso laboral. Las dificultades resultan de previsiones inadaptadas en cuanto a reglas, personal (número, formación), carga de trabajo no prevista, cambio de horarios de trenes, vías ocupadas cuando deberían de estar libres. Un aspecto sumamente importante se refiere aquí a las coordinaciones de las acciones y tomas de decisión entre el personal del centro de direccionamiento y los maquinistas. Dichas coordinaciones tienen un impacto directo en la seguridad (en color azul).

- El control en la evaluación y el diagnóstico del peligro o riesgo antes y durante la actividad laboral. El problema resulta aquí de la construcción de un diagnóstico que no corresponde a la situación actual o el no poder construirlo a tiempo. Esto puede ser el caso de uno o varios de los actores en relación con una carga de trabajo importante, un cambio en el programa previsto, en la maniobra o con una comunicación de información confusa. La consecuencia es en general una toma de decisión inadecuada, un error en la tarea, un procedimiento no adaptado : un maquinista que interpreta un gesto como une señal de "avance", un operador del centro de control del tráfico que abre una vía ocupada, etc. (en color verde).

- El control por medio de la detección del peligro. Esta detección se caracteriza aquí por la dificultad en relación con la detección de una señal del tráfico, de una maniobra que interfiere en el campo de acción, de un trayecto inadecuado o de un elemento material en la vía del tren (en color rojo).

- El control por medio de las comunicaciones entre los diferentes actores del centro de direccionamiento y maquinistas. Dos dimensiones son consideradas en esta categoría de obstáculos: por un lado lo que resulta de las comunicaciones verbales o no, directas o mediadas por el radio o teléfono; por otro lado lo que se le comunica al maquinista por medio de la señalización del tráfico. En este tipo de incidentes, la mayoría de los problemas se da por una falta de comprensión del idioma del otro, una confusión o un error en las informaciones transmitidas, la falta de información por un defecto de coordinación en el centro, la imposibilidad de interpretar o de detectar la información en un momento dado, etc. La consecuencia directa será la imposibilidad de establecer un diagnóstico correcto de la situación actual y por ende la ausencia de detección del peligro (en color rosa).

- La última categoría de análisis es la del entorno climático, geográfico y físico en relación con la infraestructura ferroviaria (tipos y números de vías, curvas, vagones que tapan un tren o una señal, lluvia, noche, señales poco visibles, cabina de la locomotora inadaptada...). Esta categoría es particular ya que algunos de los elementos de ella son fijos y otros cambian todo el tiempo. Además, la falta de luz, la lluvia o los vagones estacionados en una vía del tren en un caso aparecerán como puntos pivote pero eso no significa que estos sean factores de riesgo permanentes (en color amarillo). 
Como lo explicamos anteriormente, los puntos pivote son "pivote" en interacción con los otros y en el contexto particular del incidente. Es por eso que el diagnóstico se establece en primer lugar en base a la historia del evento y en segundo lugar en base al grupo de eventos y a la tipología.

\subsection{Cuatro tipos de eventos críticos interoperables}

El análisis permite la identificación de cuatro arquetipos de eventos críticos. Dicha clasificación se basa en los tipos de puntos pivote involucrados, sus relaciones, el modo y el momento en el cual surgen. Recordamos aquí que la tabla 2 sintetiza los puntos pivote de los 25 incidentes y ofrece un panorama global de la repartición de los incidentes y de los puntos pivote, sin tomar en cuenta la cronología de los eventos. Se aprecia entonces la frecuencia y presencia de éstos últimos según los actores involucrados, las acciones y dificultades en relación con la organización prevista y los ajustes necesarios en la situación actual en el centro de direccionamiento o la estación en la cual se produjo el incidente. A continuación se da el diagnóstico según la tipología establecida en base a esta tabla y a la historia y cronología de cada evento crítico. Con excepción de uno o dos casos, para los cuales no es fácil decidir en que arquetipo incidente se sitúan, la tipología se establece sin mayor problema.

Tipo 1 - Fallas de la comunicación entre agentes en el control del tráfico, agentes en el terreno y maquinistas (incidentes del 1 al 8, tabla 2).

Destacan aquí los problemas de comunicación entre el personal del centro de direccionamiento y los maquinistas de nacionalidad extranjera o no. La interpretación inadecuada de una información, la no comprensión de otra, inducen al maquinista en error en la mayoría de los casos, así como la omisión de una información útil al maquinista o la imposibilidad de percibir una señal en el centro de direccionamiento. En algunos casos, ciertos cambios, incidentes o problemas organizacionales se acumulan.

El maquinista no tiene o no se procura ni los elementos suficientes, ni pertinentes para establecer un diagnóstico correcto de la situación y de su evolución. En general el peligro lo percibe demasiado tarde o no lo percibe. La tabla 1 describe un ejemplo de este tipo que se produjo a principios del 2003.

El maquinista francés está en una estación alemana y espera la señal de partida por parte del "controlador [2]" alemán, que está en el andén checando la subida y bajada de los pasajeros, antes de subirse el mismo al tren.

El maquinista contacta al agente de control del tráfico [3] por radio para preguntarle si hay alguna señal aditiva de partida, pues él esperaba otra cosa. El maquinista esta parado en un lugar en donde hay una señal cerrada, lo cual significa que un incidente técnico se produjo, esto aparecerá como un elemento perturbador adicional.

Aquí es necesario explicar varios puntos : el maquinista no ha entendido que la señal está cerrada porque hay un incidente técnico; en un centro de direccionamiento alemán la partida la da el agente de control del tráfico y no el controlador, pero aquí se trata de una estación ; por último, el hecho que los franceses pasen la frontera y vayan a Alemania era extremadamente reciente en ese momento y los maquinistas decían sentirse muy nerviosos y tener varias dudas en cuanto a las reglas en Alemania.

Laboreal, Volume $1 \mathrm{~N}^{\circ} 1$ | 2005 
36 En la etapa siguiente, el agente de control del trafico le avisa que estará en contacto con un agente responsable del control de las agujas [4]. Esto induce al maquinista en error pues no ha entendido que hay un problema técnico y que por eso lo ponen en contacto con el responsable de agujas. El cree que tiene algo que ver con la información que le había pedido anteriormente al agente de control del tráfico.

El agente de las agujas le transmite una "orden A" para el incidente técnico del paso a nivel y le indica el trayecto, pero en ningún caso se trata de una "autorización de partida". La "orden A" es únicamente para informar del incidente técnico en el paso a nivel.

El maquinista no escucha la última parte de la información dada por el agente de las agujas, no entiende bien y no realiza que se trata de la "orden A". Piensa que es la autorización de partida, firma la orden en el documento correspondiente y se pasa la señal cerrada, sin autorización de partida. Su diagnóstico es erróneo.

En la etapa siguiente el agente de las agujas informa al maquinista del hecho que se pasó la señal cerrada sin autorización. El conductor no entiende lo que le dice el agente y sigue avanzando. Al llegar al paso a nivel que está descompuesto, a pesar de que las barreras se están bajando y de que un agente de la compañía ferroviaria alemana viene hacia él, el maquinista pasa el paso a nivel y toma velocidad.

El agente de control del tráfico alemán le avisa por radio que olvidó al controlador en el andén. El maquinista no sabe que hacer está sumamente perturbado.

41 Este incidente muestra los problemas de ajuste y de coordinación ante un incidente técnico, las dificultades de comunicación por radio y en idioma extranjero y la "fragilidad cognitiva" que puede favorecer y acentuar una situación crítica en un país extranjero. El maquinista se construyó un diagnóstico erróneo y no tuvo los medios cognitivos para integrar los elementos adecuados de la situación en curso, ni de procurárselos, no checa la comprensión de las informaciones comunicadas y finalmente pierde su "self-control". Este ejemplo ilustra la necesidad de montar, entre otras cosas, formaciones colectivas, involucrando tanto a los maquinistas del otro país como al personal del centro y estaciones del país que los recibirán, para el control eficaz de situaciones críticas.

Tipo 2 - Fallas en la organización de la situación en relación con la coordinación, las acciones del personal, la carga de trabajo (incidentes del 9 al 16 en la tabla 2). Se observan problemas similares a los del tipo 1 pero además dificultades organizacionales complican la situación introduciendo cambios en las acciones previstas, una carga de trabajo importante para alguno de los actores de los puestos de control del tráfico. Se advierten entonces errores como por ejemplo el dirigir un tren hacia una vía ocupada problemas en relación con una falta de formación del personal y de experiencia que fragilizan la evolución de la situación.

Tipo 3 - Fallas en la coordinación de las acciones del centro de direccionamiento (incidentes del 17 al 22 en la tabla 2).

45 En este tipo de eventos, los maquinistas son conducidos a una situación no deseada sin estar al tanto. No intervienen en el desarrollo del evento de manera directa pero son los que realizan el evento como consecuencia de las fallas en la coordinación en el centro de direccionamiento. 

puntos críticos se sitúan en las interacciones entre los actores del centro de direccionamiento y los maquinistas. Estas interacciones implican principalmente disfuncionamientos en la comunicación y en la percepción e interpretación de la señalización. Otro punto crítico de esas interacciones es la coordinación de las acciones de los actores involucrados en un proceso laboral, lo cual se agrava en ciertos casos con la carga de trabajo o la presencia de incidentes técnicos.

\subsection{Los problemas de comunicación}

52 Los problemas surgen al no entender bien a su interlocutor o entender de manera errónea una información. La consecuencia es la construcción de representaciones mentales incompatibles entre los actores basadas en un diagnóstico no adaptado a las condiciones de la situación actual. Las tomas de decisión y acciones efectuadas conducirán al evento crítico. Estos problemas de comunicación son igualmente validos entre actores que creen entenderse aún en su propio idioma. Sin embargo, en un idioma extranjero, la comprensión e interpretación de un mensaje dependen aún más del contexto en el cual se inscriben, aunque se maneje correctamente el otro idioma. Esto esta ligado el hecho de que el maquinista integra su actividad en la representación 
de la situación y en las secuencias de eventos contextualizados (Guyot, Weill-Fassina, Valot \& Amalberti, 1996). una dificultad para percibir e interpretar correctamente la señal según el punto en el cual esté el maquinista. Por ejemplo, una señal violeta que no se ve bien en la noche desde un ángulo particular, una señal que el maquinista no puede ver porque esta justo arriba de él, una señal un poco tapada por otros vagones en el centro, etc. Siete eventos críticos involucran este tipo de problemas (incidentes 4, 6, 7, 12, 17, 18 y 22).

Vinculado al problema de la visibilidad de la señalización aparece también mediante este estudio el impacto del entorno físico y meteorológico. Por ejemplo, el maquinista no tiene la posibilidad de detectar que las vías están ocupadas porque hay una curva, hay otros vagones, es de noche, esta lloviendo o nevando o el alumbrado artificial 
cambia las condiciones visuales, etc. Desde luego que cambiar estos parámetros no es posible, sin embargo se les debe tomar en cuenta de alguna manera en la organización.

61 La recomendación principal para estos puntos es entonces el integrar durante la formación de los maquinistas el conocimiento de los centros de direccionamiento que frecuentarán, efectuando practicas de campo con el fin de familiarizarlos con el futuro contexto laboral. Esto es aún más importante si se considera que la señalización es distinta en los diferentes países europeos (una misma señal puede tener un significado distinto en el otro país, una señal puede existir en uno y no en otro, las reglas de seguridad que se aplican no son exactamente las mismas para una misma señal...). Y, como pudimos observar durante nuestros estudios de campo en cuatro centros de direccionamiento, se pueden agregar dificultades de orden topológico y de orientación importante que pueden conducir a una falla por parte del maquinista si éste no conoce bien las instalaciones (De la Garza, Kaplan \& Weill-Fassina, 2003).

Además, el transporte de mercancías es principalmente un tráfico de noche por lo tanto el conocimiento de los centros de direccionamiento es un fuerte criterio de fiabilidad ya que en la noche la visibilidad es mucho más reducida. Estos parámetros se combinan probablemente con el cansancio y la vigilancia de los maquinistas que tienen turnos de noche, y aunque no abordamos ese aspecto aquí, lo señalamos pues una reflexión en este ámbito es necesaria para una visión completa de los problemas (Guyot et al., 1996).

\subsection{La organización del trabajo debe considerar y mejorar la coordinación "contextualizada"}

63 La coordinación es la regulación de la acción cooperativa, nos dice Maggi (1996) y dicha coordinación es un ordenamiento de comportamientos, acciones y decisiones. Para un funcionamiento fiable debe haber una compatibilidad y una temporalidad entre las acciones e intervenciones de los diferentes actores del centro de direccionamiento y los maquinistas. Es así como se debe distinguir la coordinación decidida antes de la acción, la cual fija las reglas de funcionamiento y orden de los trenes, etc., y la coordinación contextual que caracteriza el funcionamiento de una red de actores los cuales tienen que efectuar ajustes ante los cambios previstos o no (retardos de tren, tren antes de la hora, carga de trabajo importante, falta de personal, incidentes técnicos, etc.) (Maggi, op. cit.; De la Garza, 1999). El estudio realizado destaca que quince eventos críticos presentan problemas ligados a la coordinación de las acciones. Y curiosamente, cinco de ellos involucran únicamente al personal del centro de direccionamiento; en esos casos los maquinistas solo efectúan una orden, que es lo que conduce al incidente, pero no toman ningún tipo de decisión. Este resultado enfatiza el hecho de que independientemente de la interoperabilidad el funcionamiento fiable y eficaz de un centro de direccionamiento depende en gran medida de ésta coordinación contextual. Desde luego que para un maquinista extranjero llegar o partir de un centro de direccionamiento en el cual existen disfuncionamientos en la coordinación es un contexto propicio al error o la falla.

64 Los problemas de coordinación son entonces de dos tipos:

- coordinación de las acciones y de las ordenes dadas entre agentes de tráfico, agentes de las agujas, agentes maniobras y maquinistas. Por ejemplo al no informar al maquinista de un cambio de maniobra éste puede efectuar un trayecto inadecuado; los problemas relacionales 
entre maquinistas y agentes pueden inducir errores $u$ omisiones de transmisión de una información útil.

- coordinación de las acciones únicamente entre agentes de tráfico, agentes de las agujas, agentes maniobras. Aquí los problemas son por ejemplo que ante la carga de trabajo o la falta de experiencia de algún agente, haya olvidos o errores : un agente que olvida recuperar las agujas y ponerlas en la posición adecuada, un agente que envía un tren a una vía ocupada o un agente que no transmite una información en cuanto a una operación efectuada. Se agregan a algunas de las historias de los eventos críticos la infracción de reglas de seguridad por parte de alguno de los agentes del centro de direccionamiento, como por ejemplo el no posicionarse en donde se debe para alguna maniobra, o el no controlar correctamente alguna acción de maniobra. Sin embargo, dada la poca información contenida en los reportes, no es posible hacer un análisis detallado de esos puntos. centro de direccionamiento deben estar preparados al manejo de estas situaciones y la infraestructura ferroviaria también con el fin de facilitar la circulación hacia vías distintas según el tamaño de los trenes, las llegadas y partidas, la organización del personal del centro (por ejemplo en momentos que se saben difíciles durante el día y la noche prever más personal y prever personal capacitado). La interoperabilidad acentuará esos problemas por diferentes razones : cambio de actividades en un centro con la llegada de trenes internacionales, cambio de la carga de trabajo con el aumento según los casos de trenes que llegan a un centro. interoperable, así como de los requisitos de personal (aumentar el personal es necesario en algunos casos), es un criterio de fiabilidad para la organización futura.

\section{Conclusión}

71 La utilización de éste método como instrumento para anticipar situaciones problemáticas en un diseño futuro resulta sumamente interesante. En efecto, los eventos críticos analizados aquí no son situaciones completamente interoperables pero en las zonas fronterizas desde siempre el personal de las estaciones y centros de direccionamiento han interactuado y colaborado con los maquinistas del otro país y 
hasta con el personal de puestos de control del tráfico del otro país. La idea aquí fue la de extraer de esa experiencia situaciones críticas pudiendo conducir a un evento crítico o situaciones críticas que un maquinista extranjero tendrá que saber manejar y los elementos a nivel organizacional que deben ser previstos para que el sistema sociotécnico funcione de manera fiable y segura. Así, las recomendaciones presentadas aquí tienen por objeto final el aumentar la fiabilidad y la eficacia del sistema socio-técnico interoperable.

De acuerdo con otros autores, se insiste aquí en el hecho que el análisis de eventos críticos es un verdadero instrumento de aprendizaje para el diseño de una situación futura (Schaaf van der, Lucas \& Hale, 1991).

Estos resultados confirman también ciertos fundamentos de la fiabilidad de un sistema socio-técnico, como el hecho de que la fiabilidad humana sólo puede existir en una organización fiable que prevé los recursos necesarios en términos de material, de equipo, de personal, de formación, de reglamentos, de tiempo, etc. (De la Garza, 1999 ; Reason, 2004).

El análisis de eventos críticos pone en relieve el rol activo y directo de toda una red de actores involucrados en los procesos laborales y por ende en los eventos críticos. La reflexión en cuanto a la seguridad debe considerar esta red de actores y las coordinaciones que la caracterizan así como los requisitos para un funcionamiento fiable de éstas. El considerar únicamente al maquinista en la organización de la interoperabilidad sería un error y una visión parcial del sistema socio-técnico. La anticipación consiste en saber a través del análisis de estos incidentes el tipo de problemas con los cuales se enfrentarán los maquinistas extranjeros y el personal de un centro de direccionamiento y de una estación de tren en Francia y probablemente en otros países europeos. La diferencia principal siendo que los actores en los centros de direccionamiento pueden tener la misma función o estatuto y ejercer tareas distintas, o puede haber alguna función que existe en un país y no en el otro, etc. Los factores identificados como puntos pivote pueden surgir en una situación sin interoperabilidad o con interoperabilidad, pues como lo dijimos anteriormente, no son todos exclusivos de una situación interoperable. Pero, con la llegada de la interoperabilidad algunos de estos puntos críticos pueden acentuarse. Desde luego que no pretendemos que nuestro estudio sea exhaustivo y es probable que otros puntos-pivote puedan aparecer al momento de la interoperabilidad total y efectiva. Sin embargo en el 2003 y el 2004 no se pudo obtener información en cuanto a los incidentes que se han producido en el eurostar, que es un caso de interoperabilidad total.

Las recomendaciones finales fueron presentadas en forma de un instrumento metodológico el cual es divulgado por la Unión Internacional de Ferrocarriles (De la Garza, et al., 2004). Dicho instrumento fue validado por la Comisión de Energía y Transportes de la Unión Europea y está a disposición de las empresas que desean organizar un servicio interoperable.

Amalberti, R. (2001). The paradoxes of almost totally safe transportation systems. Safety Science, 37, 109-126.

77 Cuq, J.P., \& Gruca, I. (2002). Cours de didactique du français en langue étrangère et seconde. PUG : Grenoble. 
 (2004). Building a safe, interoperable railway: a methodological guide to integrating human factors. Université Paris 5- EPHE - TU Berlin - UIC. Document published by the UIC. UIC : Paris.

Fadier, E., De la Garza, C., \& Didelot, A. (2003). Safe design and human activity: construction of a theoretical framework from an analysis of a printing sector. Safety Science , 41, (9), 759-789.

Guyot, S, Weill-Fassina, A., Valot, Cl., \& Amalberti, R. (1996). Gestion des contraintes de travail par les Agents de conduite. Rapport final. Contrat LEPC EPHE/ CERMA/ SNCF / NEB. Paris : EPHE-CERMA.

Guyot-Delacroix, S. (2002). Aux frontières de l'adaptation: conditions et circonstances des “échecs» de régulation de l'activité. L'exemple de la conduite des trains. In R. Amalberti, C. Fuchs, \& C. Gilbert (S/ D), Risques, erreurs et défaillances. Approche interdisciplinaire (pp. 319-335). Grenoble : Publications de la MSH-Alpes, CNRS.

Hale, A.R., \& Glendon, A.I. (1987). Individual behaviour in the control of danger. Amsterdam : Elsevier Sc. Publishers .

Leplat, J., \& de Terssac, G. (1990). Les facteurs humains de la fiabilité dans les systèmes complexes. Marseille : Octarès.

Maggi, B. (1996). La régulation du processus de travail. In P. Cazamian, F. Hubault, \& M. Noulin, Traité d'ergonomie (3ème édition) (pp. 637662). Toulouse : Octarès.

Perrow, C (1984). Normal accidents, Living with High-Risk Technologies. New York: Basic Books, Inc. Publishers.

Rasmussen, J (1997). Risk management in a dynamic society : a modelling problem. Safety Science 27 (2/3), 183-213.

90 Reason, J. (1990). Human Error. Cambridge : University Press.

Reason, J. (2004). Managing the risks of organizational accidents. Burlington : Ashgate publishing limited.

Schaaf van der, T.W., Lucas, D. \& Hale, A.R. (eds.) (1991). Near miss reporting as a safety tool. Oxford: Butterworth Heinemann Ltd.

Weill-Fassina, A., De la Garza, C., \& Kaplan, M. (2003). Analyse de situations événementielles. Dossier 3, Etude interopérabilité Fret France-Allemagne, Contrat UICNEB-INEREC. Paris : UIC EPHE Paris 5. 
Tabla 1 Reconstituición de la historia del incidente interoperable $n^{\circ} 2$ en base a la tabla de análisis general para todos los incidentes de éste tipo

\begin{tabular}{|c|c|c|c|c|c|c|c|c|c|c|}
\hline \begin{tabular}{|l|} 
Cronologia \\
\end{tabular} & $\mathrm{T}_{1}$ & $T_{2}$ & $\mathrm{~T}_{3}$ & $T_{4}$ & IS & T6 & T7 & 18 & T9 & $T_{10}$ \\
\hline 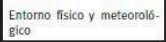 & $\begin{array}{l}2 \text { a futimos vago- } \\
\text { nes en el anden }\end{array}$ & & & & & & & & & \\
\hline Organizacíion del trabajio & & & & & & & & & & \\
\hline \multirow{2}{*}{ 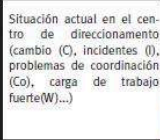 } & 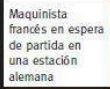 & 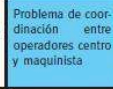 & 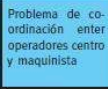 & & & 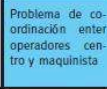 & & 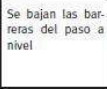 & & \\
\hline & 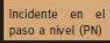 & & & & & & & & & \\
\hline 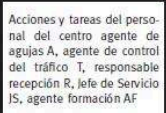 & & & 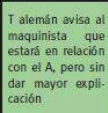 & 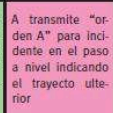 & & 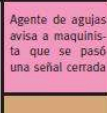 & & & & 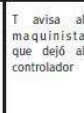 \\
\hline 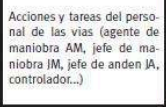 & 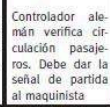 & & & & & & & & & \\
\hline \multirow[t]{2}{*}{ 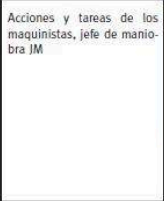 } & 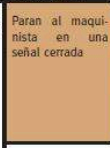 & 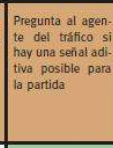 & $\begin{array}{l}1 \text { maquinista no } \\
\text { entende bien }\end{array}$ & 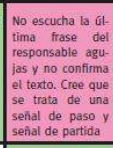 & 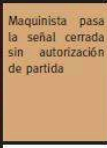 & & \begin{tabular}{|l} 
No entiende biven \\
al agente de agu- \\
ass
\end{tabular} & 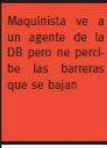 & 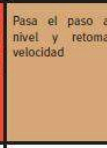 & 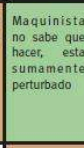 \\
\hline & & 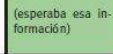 & & 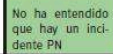 & & & & & & \\
\hline 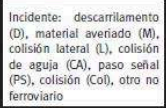 & & & & & ps & & & & & 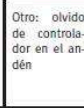 \\
\hline
\end{tabular}

Tabla 2 Tabla de los puntos pivote en relación con cada uno de los 25 incidentes interoperables

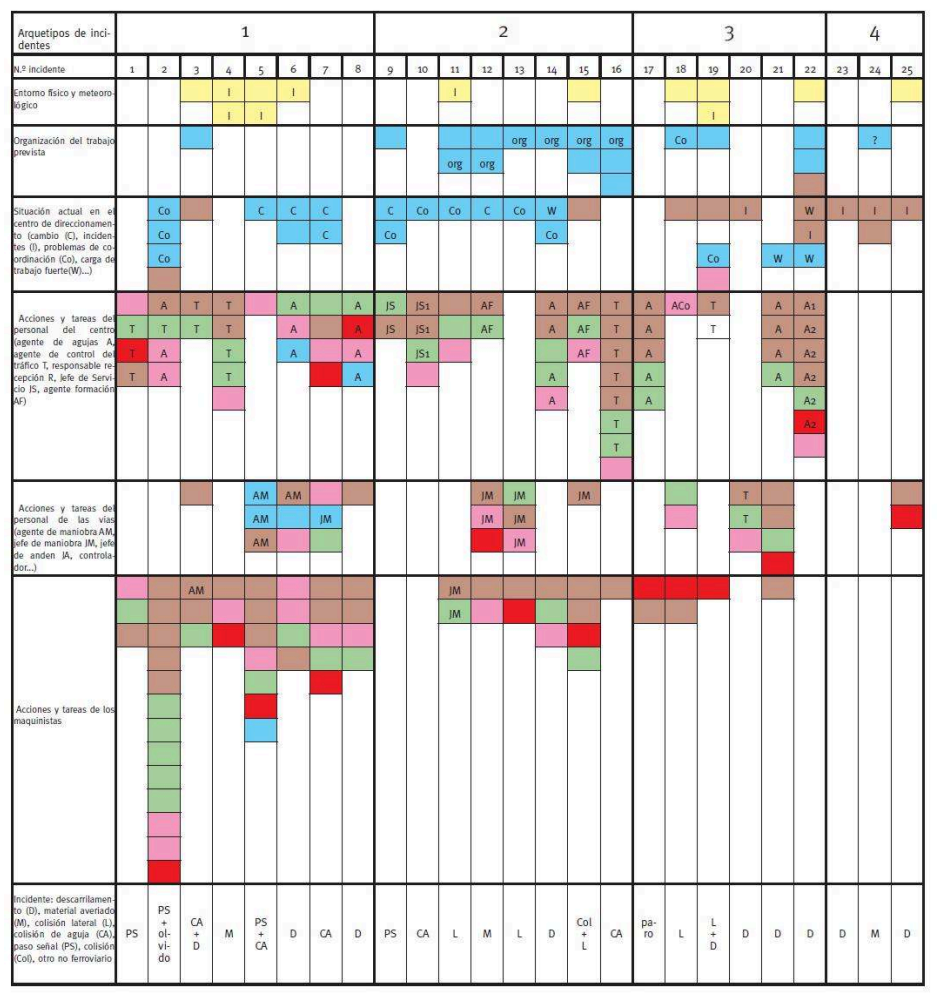




\section{ANEXOS}

Anexo 1 Behaviour in the face of danger model (Hale \& Glendon, 1987)

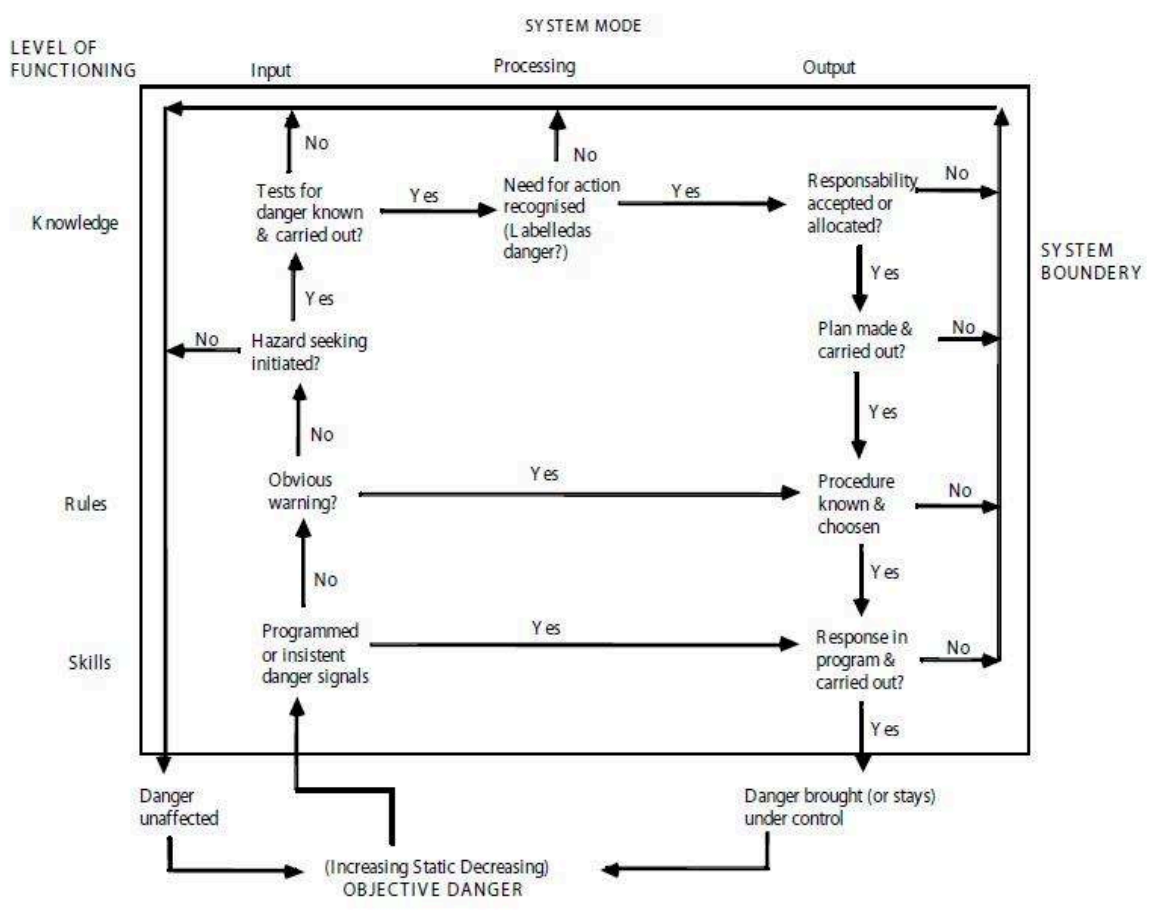

\section{NOTAS}

1. Equipo francés: De la Garza C., Universidad Descartes Paris 5, WeillFassina A., Kaplan M., École Pratique des Hautes Études. Equipo alemán: Wilpert B., Schmidt A., Fhalbruch B., Universidad Técnica de Berlín.

2. El controlador es un agente de la compañía de ferrocarriles alemana cuyo rol es, antes de la partida del tren, él de informar a los pasajeros, checar que los pasajeros suban al tren, y que no haya nadie al momento de cerrar las puertas y dar la partida al maquinista. Un vez el tren en camino, el controlador checa los boletos de tren de cada uno de los pasajeros durante el viaje.

3. En Alemania éste agente no se mueve de su puesto de control del tráfico y comunica únicamente por radio con los maquinistas.

4. En Alemania éste agente se ocupa del control únicamente de un grupo de agujas en una estación o centro de direccionamiento y también comunica únicamente por radio con los maquinistas. 


\section{RESÚMENES}

En un programa europeo, se presenta un estudio de veinticinco eventos críticos teniendo por objetivo demostrar cómo un método de análisis de accidentes podría contribuir a la seguridad en la concepción de una organización futura. Dichos eventos se consideran interoperables ya que implican una locomotora o un maquinista extranjero en una zona fronteriza francesa. El método de los "puntos pivote" se caracteriza por cuatro etapas que permiten combinar al análisis de la actividad laboral, el análisis de los reportes de eventos, el análisis cognitivo de las acciones y diagnósticos que explican, en interacción con las condiciones de trabajo, la emergencia de un evento crítico. Se pudo entonces explicar la emergencia de cada incidente y establecer cuatro tipos de incidentes. Estos últimos mostrando principalmente problemas de comunicación en una lengua extranjera, de comunicación e interacción con la señalización ferroviaria y dificultades de coordinación de las acciones y decisiones entre los actores de una red compleja (agentes del centro de direccionamiento y de las estaciones y los maquinistas. La conclusión destaca una serie de recomendaciones para la organización futura de una interoperabilidad segura integrando los riesgos identificados aquí. Además de las barrera técnicas y reglas de seguridad, se trata de barreras organizacionales las cuales garantizan de cierto modo la fiabilidad humana y la eficacia de un sistema socio-técnico.

No âmbito de um programa europeu, apresenta-se um estudo de vinte e cinco acontecimentos críticos tendo por objectivo demonstrar como um método de análise de acidentes poderia contribuir para a segurança na concepção segura de uma organização futura. Tais eventos são considerados interoperáveis já que implicam uma locomotiva ou um maquinista estrangeiro numa zona fronteiriça francesa. 0 método dos "pontos-pivot" caracteriza-se por quatro etapas que permitem combinar a análise da actividade de trabalho, a análise dos relatórios de acontecimentos, a análise cognitiva das acções e diagnósticos que explicam, em interacção com as condições de trabalho, a emergência de um acontecimento crítico. Pode-se então explicar a emergência de cada incidente e estabelecer quatro tipos de incidentes. Estes últimos mostrando principalmente problemas de comunicação numa língua estrangeira, de comunicação e interacção com a sinalização ferroviária e dificuldades de coordenação das acções e decisões entre os actores de uma rede complexa (agentes do centro de direccionamento e das estações e os maquinistas). A conclusão destaca uma série de recomendações para a organização futura de uma interoperabilidade segura, integrando os diferentes riscos aqui identificados. Além das barreiras técnicas e regras de segurança, trata-se de barreiras organizacionais, as quais garantem de certo modo a fiabilidade humana e a eficácia do sistema sócio-técnico.

Dans le cadre d'un projet européen, une étude de vingt-cinq événements critiques avait pour but de montrer comment une méthode d'analyse d'accidents pouvait contribuer à la conception sûre d'une future organisation. Ces événements sont considérés “interopérables", puisqu'ils impliquent un conducteur ou une locomotive étrangère dans une zone frontalière en France. La méthode des “points-pivots » utilisée ici, comprend quatre étapes qui permettent d'articuler l'analyse de l'activité de travail, l'analyse de rapports d'événements critiques et l'analyse cognitive des actions et diagnostics en interaction avec les conditions de travail. Ainsi on a pu expliquer l'émergence de chaque incident et établir quatre types d'incidents. Ces derniers montrent principalement des problèmes de communication dans une langue étrangère, de communication et d'interaction avec la signalisation ferroviaire et des difficultés de coordination des actions et décisions entre les acteurs d'un réseau complexe (agents du centre de triage et gares et conducteurs). La conclusion fait ressortir une série de recommandations pour l'organisation future sûre intégrant les divers risques identifiés ici. Au-delà de barrières 
techniques et des règles de sécurité, des barrières organisationnelles sont des vrais garants de la fiabilité humaine et de la performance du système socio-technique.

In a European project, a study of 25 critical events was conducted in order to show how an accident analysis method could contribute to the safe design of a future organisation. These events are "inter-operable" because they involved a foreign driver or a foreign locomotive in a French border zone. The "pivotal-point" method, used here, requires four steps that connect the work activity analysis, the accident report analysis and the cognitive analysis of the actions and diagnostics, in interaction with the work environment. So, it is possible to explain how incidents occurred and to establish a typology. Four kinds of inter-operable incidents highlighted that the difficulties are linked to communication in a foreign language; the communication and the interaction with the signalling system ; and the coordination of the actions and decision-making between various actors of a complex network (the marshalling yard personnel and the drivers). To conclude, some recommendations are given for the design of a safe future organisation taking into account the risks and difficulties identified here. In fact, beyond the technical barriers and safety regulations, real organisational barriers should be considered to guarantee the human reliability and the performance of the socio-technical system.

\section{ÍNDICE}

Palabras claves: diseño seguro, análisis cognitivo, análisis incidente, interoperabilidad ferroviaria, fiabilidad

Keywords: safe design, cognitive analysis, incident analysis, railway interoperability, reliability Palavras-chave: concepção segura, análise cognitiva, análise de incidentes, interoperabilidade ferroviária, fiabilidade

Mots-clés: conception sûre, analyse cognitive, analyse incident, interopérabilité ferroviaire, fiabilité

\section{AUTOR}

\section{CECÍLIA DE LA GARZA}

Universidad Descartes Paris 5, Laboratorio de Ergonomía Informática, 45, rue des Saints-Pères, 75006 Paris - France

cecilia.de-la-garza-corona@univ-paris5.fr 